\title{
Première Caractérisation Pomologique Des Variétés Locales De L'olivier (Olea Europaea L.) Des Oliveraies Traditionnelles Des Agroécosystèmes Des Montagnes Du Nord-Ouest Du Maroc
}

\author{
Hicham Barbara, \\ Laboratoire Botanique Appliquée - Equipe BioAgrodiversité \\ Université Abdelmalek Essaâdi \\ Institut des Sciences de l'Evolution de Montpellier (ISE-M | UMR 5554). \\ Equipe Dynamique de la Biodiversité, Anthropo-écologie \\ Laboratoire International Associé Evolea (INEE-CNRS /CNRST) \\ Jean-Frederic Terral, \\ Institut des Sciences de l'Evolution de Montpellier (ISE-M | UMR \\ 5554).Equipe Dynamique de la Biodiversité, Anthropo-écologie \\ Laboratoire International Associé Evolea (INEE-CNRS /CNRST)

\section{Mohammed Ater,} \\ Laboratoire Botanique Appliquée- Equipe BioAgrodiversité \\ Université Abdelmalek Essaâdi \\ Laboratoire International Associé Evolea (INEE-CNRS /CNRST)
}

Doi:10.19044/esj.2020.v16n6p556 ～URL:http://dx.doi.org/10.19044/esj.2020.v16n6p556

Résumé

Les oliveraies traditionnelles du nord-ouest du Maroc se différencient par une diversité variétale relativement importante contrairement au paysage oléicole marocain dominé par une seule variété, la Picholine marocaine. En effet, les pratiques traditionnelles locales à travers le maintien des vergers poly-variétaux ont permis la conservation de variétés locales rares et sousutilisées. Dans cette étude nous avons réalisé l'échantillonnage selon un dispositif équilibré (10 arbres par variété et 30 olives par arbre) des 6 variétés locales dénommées et reconnues par les agriculteurs (Zeitoun, Bouchouk, Hamrani, Kortbi, Asemlal, Semlal), d'un écotype d'oléastre forme spontanée de l'olivier et deux variétés sélectionnées utilisées par les agriculteurs (Haouzia et Menara). L'étude pomologique s'est appuyée sur l'utilisation de 15 caractères quantitatifs décrivant la taille et la forme des olives et des noyaux ainsi qu'une estimation indirecte de la teneur en huile. Les résultats obtenus montrent que les variétés étudiées se différencient le long d'un gradient de taille et du potentiel productif, les caractères relatifs à la forme jouent un rôle 
secondaire dans cette différentiation. Ainsi, les variétés à petites olives (Oléastre et Asemlal) se différencient nettement des variétés à grosses olives (Meslal) et des variétés à olives de tailles intermédiaires (Zeitoun, Hamrani, Bouchouk, Kortbi, Haouzia et Menara). Les variétés de tailles intermédiaires se différencient plutôt par les caractères relatifs à la forme. La variété Kortbi se différencie nettement en s'opposant aux variétés sélectionnées Haouzia et Menara. Les variétés Zeitoun, Bouchouk et Hamrani se chevauchent largement.

Mots clés: Olivier, Variétés locales, Nord-ouest Maroc, diversité, oliveraies traditionnelles

\title{
First Pomological Characterization of Local Varieties of the Olive Tree (Olea Europaea L.) of the Traditional Olive Orchads of the Agroecosystems of the Mountains of North-West Morocco, an Neglected Agrobiodiversity to Preserve
}

\author{
Hicham Barbara, \\ Laboratoire Botanique Appliquée - Equipe BioAgrodiversité \\ Université Abdelmalek Essaâdi
}

Institut des Sciences de l'Evolution de Montpellier (ISE-M | UMR 5554).

Equipe Dynamique de la Biodiversité, Anthropo-écologie

Laboratoire International Associé Evolea (INEE-CNRS /CNRST)

Jean-Frederic Terral,

Institut des Sciences de l'Evolution de Montpellier (ISE-M | UMR

5554).Equipe Dynamique de la Biodiversité, Anthropo-écologie

Laboratoire International Associé Evolea (INEE-CNRS /CNRST)

Mohammed Ater,

Laboratoire Botanique Appliquée- Equipe BioAgrodiversité

Université Abdelmalek Essaâdi

Laboratoire International Associé Evolea (INEE-CNRS /CNRST)

Abstract

Unlike the Moroccan olive oil landscape characterized by the dominance of a single variety, the Moroccan Picholine, the traditional olive groves of north-west Morocco are distinguished by a relatively large variety 
diversity. Indeed, local traditional practices through the maintenance of polyvarietal orchards have allowed the conservation of rare and underused local varieties. In this study we carried out the sampling according to a balanced design (10 trees per variety and 30 olives per tree) of the 6 local varieties named and recognized by the farmers (Zeitoun, Bouchouk, Hamrani, Kortbi, Asemlal, Semlal), an oleaster spontaneous form of the olive tree and two selected varieties used by farmers (Haouzia and Menara). The pomological study was based on the use of 15 quantitative characters describing the size and shape of the olives and endocarp, as well as an indirect estimate of the oil content. The results obtained show that the varieties studied are differentiated along a size gradient and the productive potential, the characters relating to the form play a secondary role in this differentiation. The varieties with small olives (Oleaster and Asemlal) are clearly distinct from the varieties with large olives (Meslal) and the olive varieties of intermediate sizes (Zeitoun, Hamrani, Bouchouk, Kortbi, Haouzia and Menara). The varieties of intermediate sizes are rather differentiated by the characters relating to the form. The Kortbi variety is clearly differentiated by opposing the selected Haouzia and Menara varieties. The varieties Zeitoun, Bouchouk and Hamrani largely overlap.

Keywords: Olive tree, Local varieties, Northwest Morocco, diversity, Traditional olive orchads

\section{Introduction}

L'olivier représente la principale espèce fruitière au Maroc où il occupe des superficies importantes, plus de 560000 ha (El Mouhtadi et al., 2014). En effet, l'oléiculture connaît actuellement avec le «Plan Maroc Vert » une extension soutenue des superficies oléicoles visant d'atteindre 1220000 ha à l'horizon 2020 (MAPM, 2008). La conséquence de cette dynamique menant à la modernisation de la filière oléicole est l'accentuation de la marginalisation des filières oléicoles traditionnelles. Cependant, il ne faut pas oublier l'importance socio-économique et territoriale de ces filières et des oliveraies traditionnelles qui couvrent de larges territoires reconnus comme des «Zones d'agriculture solidaire» (Kradi, 2012). Il s'agit principalement d'agroécosystèmes traditionnels des zones montagneuses, comme c'est le cas dans le nord du Maroc (Hmimsa et al., 2008, Ater et al., 2013).

L'oléiculture pratiquée dans ces agroécosystèmes est extensif et pluvial. Le type extensif et dispersé des plantations favorise la pratique des polycultures associées à l'élevage. Ainsi, en plus d'autres fruitiers, les paysans cultivent des céréales ou des légumineuses sur les mêmes parcelles. Ce type d'oliveraies s'apparente plus à un système agroforestier qu'à un verger fruitier au sens strict du terme (Lauri et al., 2019). D'autre part, les formes spontanées de l'olivier sont exploitées par les agriculteurs. Ces formes spontanées ou 
oléastres sont dénommées localement «Berri » et peuvent correspondre soit à des formes sauvages de l'olivier (Olea europaea subsp. euroapea var. sylvestris), soit à des formes férales échappées des cultures et issues de semis d'oliviers cultivés. Dans les oliveraies traditionnelles, l'oléastre est utilisé principalement comme porte greffe, mais il peut aussi être utilisé pour la production de 1'huile (Aumeeruddy-Thomas et al., 2014, 2017, Ater et al., 2016, Adim, 2018). Du point de vue diversité variétale, les oliveraies du nord du Maroc sont dominées par la variété «Zeitoun» ou «Zeitoun Beldi », ce qui n'empêche pas la conservation des variétés locales par la pratique courante de vergers poly-variétaux (Ater et al., 2016). En effet, plusieurs travaux ont noté l'existence d'une diversité variétal importante dans les oliveraies traditionnelles du Nord du Maroc (Haouane, 2012; Kartas et al., 2015; Kartas, 2017). Il faut noter que la variété locale «Zeitoun » correspond à ce que les agronomes appellent la «Picholine marocaine» variété dominante au Maroc et dénommée «Sigoise » en Algérie (Boulouha et al., 1992 ; Khadari et al., 2008, 2016; Moukhli et al., 2013, 2016).

Ainsi, il apparaît clairement que l'oléiculture traditionnelle dans les agroécosystèmes des montagnes du nord du Maroc présente des caractéristiques importantes, uniques et rares à prendre en considération dans le contexte actuel des changements globaux (climatiques, socio-économiques, politiques agricoles,..): (1) le caractère agroforestier des oliveraies traditionnelles bien adaptées au contexte socio-économique et environnemental qui leur confère la résilience nécessaire pour faire face aux changements, (2) l'existence d'une agrobiodiversité représentée par les variétés locales (écotypes, cultivars) sous-utilisées et négligées correspondant à des ressources génétiques permettant d'élargir la base génétique de l'olivier. Dans ce travail, nous nous intéressons particulièrement à la diversité variétale sous-utilisée à travers la caractérisation des variétés locales des oliveraies traditionnelles du Nord-ouest du Maroc. Ainsi, en se basant sur des inventaires et des enquêtes antérieures sur l'agrobiodiversité dans les agroécosystèmes traditionnels du nord du Maroc (Ater et al., 2016), nous réalisons une caractérisation pomologique par une étude morphométrique traditionnelle des olives et des noyaux des variétés locales recensées dans la région. Il s'agit des variétés nommés localement Zeitoun, Hamrani, Bouchouk, Asemlal, Meslal et Kortbi. En plus de ces variétés, nous avons inclus l'oléastre dans cette étude, eu égard à son importance dans les pratiques traditionnelles ainsi que deux variétés sélectionnées récemment (Haouzia et Menara), distribuées aux agriculteurs par les services du Ministère de l'Agriculture dans le cadre des programmes d'appui au développement de la filière oléicole. 


\section{Matériel et méthodes}

Aire d'étude : La zone d'étude correspond à la Péninsule Tingitane au Nord-Ouest du Maroc. Du point de vue géographique, la zone occupe la majeure partie de la chaîne du Rif occidental. C'est un territoire caractérisé par un relief accidenté avec des pentes fortes à l'exception des zones périphériques orientées vers l'intérieur du pays. Du point de vue agroécologique, ce territoire se situe dans une zone favorable à pluviométrie supérieure à $450 \mathrm{~mm}$ (Balaghi et al., 2013). Les agroécosystèmes de la région sont caractérisés par la coexistence d'un système sylvo-pastoral et d'une activité agricole de subsistance et forment des unités paysagères particulières. Les principales caractéristiques des agroécosystèmes de la zone d'étude sont: une agriculture traditionnelle à caractère vivrier, la pratique de la polyculture, la prédominance de la micro ( superficie $<0.5$ ha) et de la petite propriété (superficie < 5 ha), la présence de la culture du chanvre (Cannabis sativa), une large utilisation des variétés locales et un savoir-faire local traditionnel vivant (Ater et al., 2013).

L'échantillonnage a été réalisé dans six localités (Mwawja, El Achayech, Chaouen, Mesmouda, Mjaâra et Siwana) localisées dans les provinces de Tétouan, Chaouen, Ouezzane et Taounate (Fig. 1 et Tableau 1).

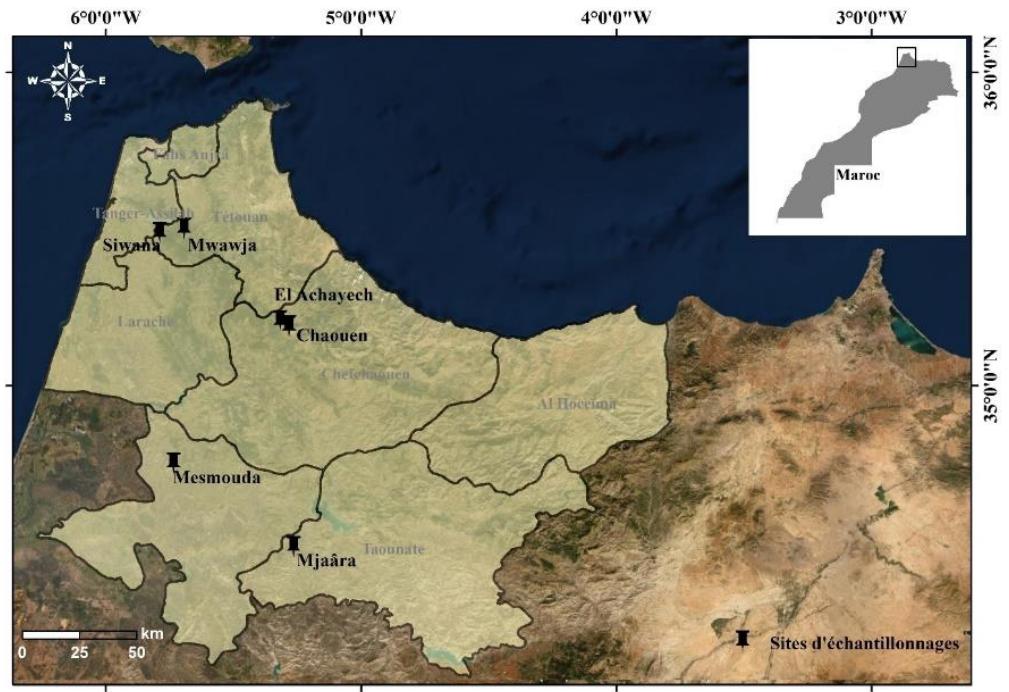

Figure 1. Carte de localisation des sites d'échantillonnage avec la délimitation des provinces. (Source, auteurs) 
Tableau 1. Coordonnées des vergers où l'échantillonnage a été réalisé avec les variétés échantillonnées par site.

\begin{tabular}{|c|c|c|}
\hline Sites & Coordonnées géographiques & Variétés échantillonnées \\
\hline Chaouen & $35^{\circ} .10 .782^{\prime} \mathrm{N} ; 005^{\circ} 16.960^{\prime} \mathrm{W}$ & Zeitoun \\
\hline El Achayech & $35^{\circ} .12 .409^{`} \mathrm{~N} ; 005^{\circ} 19.011^{\prime} \mathrm{W}$ & $\begin{array}{c}\text { Hamrani, Bouchouk, } \\
\text { Meslal }\end{array}$ \\
\hline Mesmouda & $34^{\circ} .45 .001{ }^{\prime} \mathrm{N} ; 005^{\circ} 44.039^{\prime} \mathrm{W}$ & Assemlal \\
\hline Mwawja & $35^{\circ} .30 .163^{`} \mathrm{~N} ; 005^{\circ} 41.558^{\prime} \mathrm{W}$ & Kortbi \\
\hline Mjaâra & $34^{\circ} .51 .673{ }^{\prime} \mathrm{N} ; 005^{\circ} 33.298^{\prime} \mathrm{W}$ & Menara et Haouzia \\
\hline Siwana & $35^{\circ} .29 .339^{`} \mathrm{~N} ; 005^{\circ} 47.348^{\prime} \mathrm{W}$ & Oléastre \\
\hline
\end{tabular}

Echantillonnage : L'échantillonnage des variétés a été réalisé dans des oliveraies traditionnelles en présence des agriculteurs pour s'assurer de l'identification des variétés et des noms utilisés localement. Ainsi, 6 variétés locales ont été échantillonnées et correspondent selon les dénominations utilisées à : Zeitoun, Hamrani, Bouchouk, Meslal, Kortbi, Assemlal. Nous avons également échantillonné dans le même type de vergers, deux variétés sélectionnées (Menara et Haouzia). L'oléastre a été échantillonné dans un écosystème forestier de type matorral. Pour chaque variété, nous avons échantillonné 10 arbres et pour chaque arbre, nous avons prélevé 30 fruits qui feront l'objet de la caractérisation pomologique et morphométrique.

Caractères mesurés : Les caractères mesurés permettent une évaluation quantitative de trois aspects du fruit et du noyau concernant la forme, la taille et des indicateurs sur la teneur en huile (Tab. 2). Ainsi nous avons mesuré : la longueur, la largeur et la hauteur de la plus grande largeur des olives et des noyaux à l'aide d'un pied à coulisse avec une précision de $10^{-2} \mathrm{~mm}$. La masse a été mesurée avec $10^{-4} \mathrm{~g}$ de précision. D'autres caractères ont été estimés par des rapports entre caractères mesurés (Tab. 2). 
Tableau 2. Liste des caractères mesurés ou calculés avec les codes correspondant

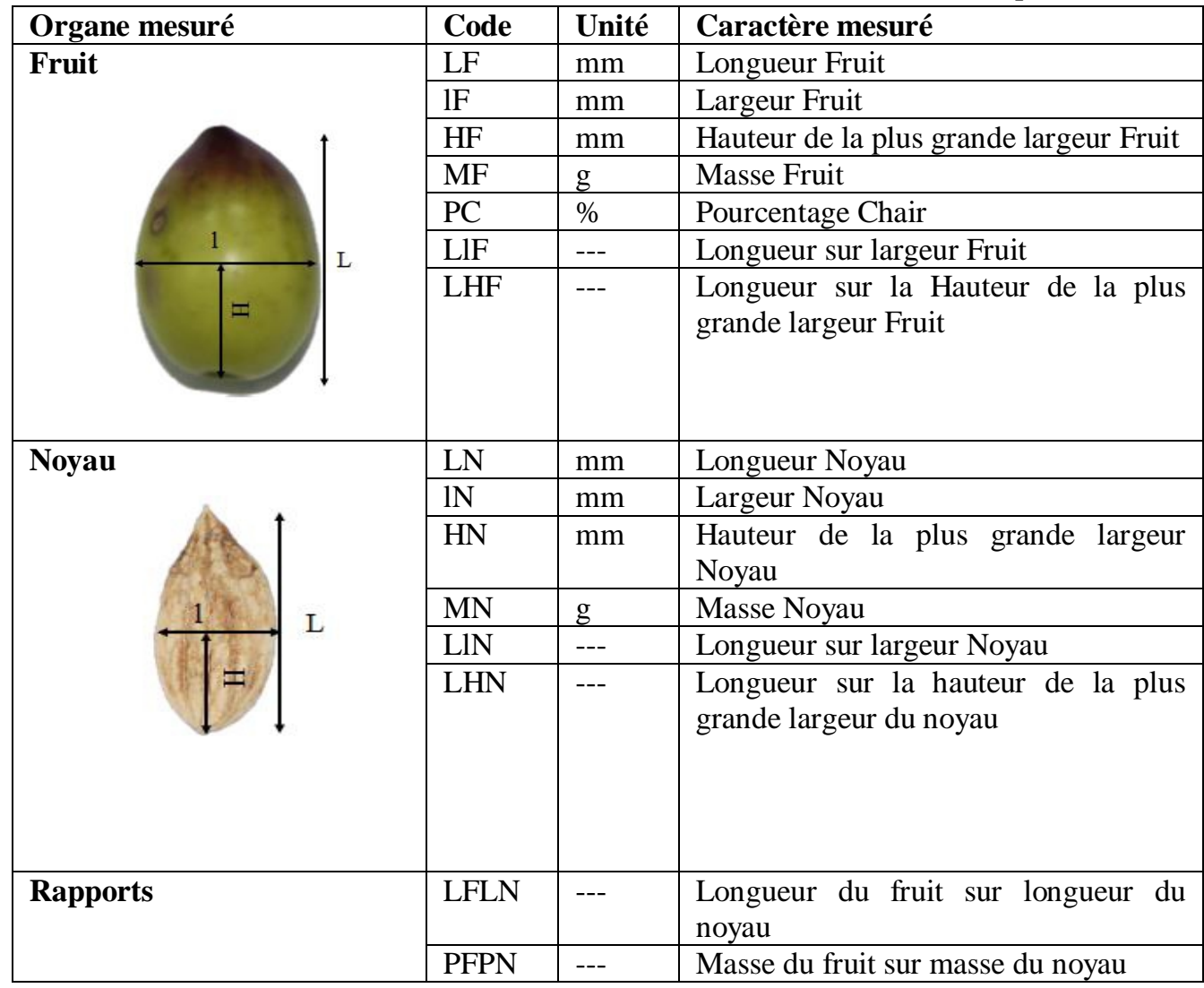

Analyses des données : Pour standardiser les variances, les variables relatives au poids et longueurs ont été transformées en $\log _{10}$. Pour la décomposition de la variance nous avons utilisé le logiciel R. Les analyses multivariées (Analyse hiérarchique (classification hiérarchique ascendante) et Analyse Factorielle Discriminante) ont été réalisées avec le logiciel XLSTAT.

\section{Résultats et discussions}

Les variétés ont été échantillonnées dans des conditions pédoclimatiques et des pratiques culturales similaires. Elles peuvent être classées en trois groupes :

1) Les variétés locales reconnues par une dénomination locale et dont les vergers sont formés d'arbres obtenus par les méthodes traditionnelles de multiplication pratiquées par les agriculteurs, souvent par greffage sur l'oléastre (Zeitoun, Hamrani, Bouchouk, Meslal et Kortbi), sauf la variété Assemlal issues de semis.

2) Les variétés sélectionnées inscrites au catalogue et multipliées par les pépiniéristes (Haouzia et Menara). 
3) L'oléastre correspondant à l'olivier spontané et que nous pouvons trouver aussi bien dans les agrosystèmes que dans les écosystèmes forestiers naturels.

Ces variétés ont des usages différents : Meslal est utilisée comme olive de table, Asemlal et l'oléastre sont utilisés pour l'huile et le reste des variétés ont un double usage.

Comme nous pouvons l'observer, ces variétés présentent des olives de tailles et formes différentes dont la caractérisation morphologique quantitative (analyse pomologique) est présentée dans cette partie (Fig. 2). Les noyaux sont également caractérisés par un important polymorphisme morphologique (Fig. 3). L'approche biométrique nous permettra de caractériser la différenciation de ces variétés à travers des descripteurs ou caractères quantitatifs. Les caractères utilisés dans cette étude (Tab. 2) sont inspirés des descripteurs utilisés par le COI (1997). On peut les diviser en trois types : i) des caractères permettant de décrire la taille de l'olive et du noyau à travers la longueur (LF et $\mathrm{LN})$, la largeur $(\mathrm{IF}, \mathrm{IN})$ et le poids $(\mathrm{PF}, \mathrm{PN})$; ii) des caractères permettant de décrire la forme et correspondent à l'allongement du fruit et du noyau estimée par le rapport de la longueur sur la largeur ; à la forme (elliptique, ovée ou obovée) estimée par la hauteur de la plus grande largeur et son rapport avec la longueur.

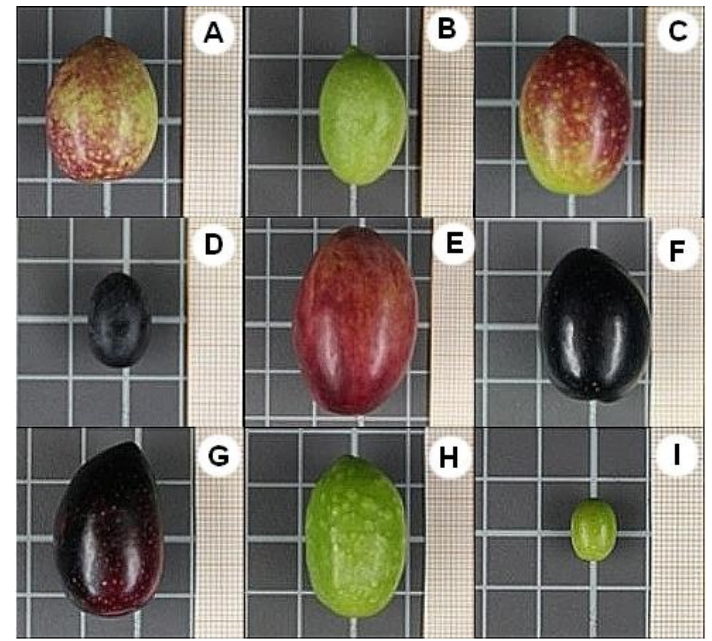

Figure 2. Olives des variétés échantillonnées : Hamrani (A), Bouchouk (B), Zeitoun (C), Assemlal (D), Meslal (E), Menara (F), Haouzia (G), Kortbi (H), Oléastre (I). (Crédit photo, auteurs). 


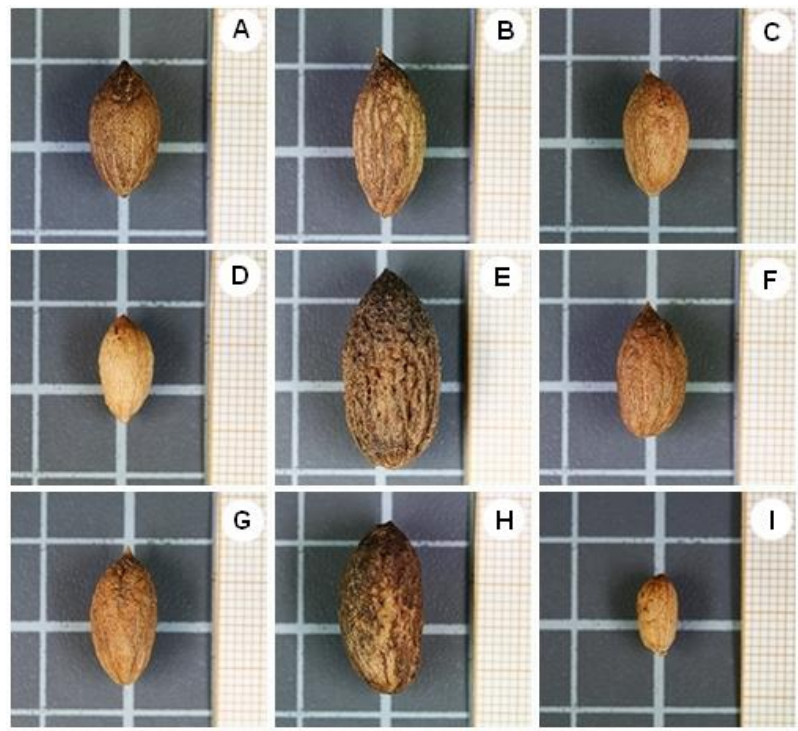

Figure 3. Noyaux des olives des différentes variétés échantillonnées : Hamrani (A), Bouchouk (B), Picholine (C), Assemlal (D), Meslal (E), Menara (F), Haouzia (G), Kortbi (H), Oléastre (I). (Crédit photo, auteurs).

\section{Caractères relatifs à la taille :}

Que ce soit pour le fruit ou pour le noyau, les différences de taille entre les variétés peuvent être appréciées par les valeurs moyennes des caractères (LF, IF, MF, LN, IN, MN) (Tab. 3).

Tableau 3 : Valeurs moyennes avec intervalle de confiance à $\mathrm{P}<0,05$ pour les caractères relatifs à la taille.

\begin{tabular}{|c|c|c|c|c|c|c|}
\hline Variétés & LF & IF & MF & $\mathbf{L N}$ & IN & MN \\
\hline Zeitoune & $21,35 \pm 0,20$ & $16,23 \pm 0,12$ & $3,44 \pm 0,08$ & $16,30 \pm 0,18$ & $8,05 \pm 0,06$ & $0,53 \pm 0,01$ \\
\hline Hamrani & $22,77 \pm 0,22$ & $17,90 \pm 0,15$ & $4,39 \pm 0,40$ & $16,25 \pm 0,18$ & $8,56 \pm 0,06$ & $0,62 \pm 0,01$ \\
\hline Bouchouk & $21,62 \pm 0,23$ & $15,09 \pm 0,16$ & $2,95 \pm 0,08$ & $16,71 \pm 0,17$ & $8,08 \pm 0,06$ & $0,53 \pm 0,01$ \\
\hline Kortbi & $24,63 \pm 0,31$ & $16,97 \pm 0,22$ & $4,39 \pm 0,16$ & $19,13 \pm 0,23$ & $9,55 \pm 0,11$ & $0,95 \pm 0,03$ \\
\hline Meslal & $29,42 \pm 0,41$ & $22,97 \pm 0,29$ & $8,72 \pm 0,29$ & $20,79 \pm 0,27$ & $10,48 \pm 0,11$ & $1,09 \pm 0,03$ \\
\hline Assemlal & $15,05 \pm 0,65$ & $10,53 \pm 0,59$ & $1,12 \pm 0,14$ & $12,84 \pm 0,56$ & $6,32 \pm 0,19$ & $0,26 \pm 0,02$ \\
\hline Menara & $25,12 \pm 0,24$ & $18,61 \pm 0,13$ & $4,99 \pm 0,10$ & $17,85 \pm 0,19$ & $8,46 \pm 0,06$ & $0,67 \pm 0,02$ \\
\hline Haouzia & $24,91 \pm 0,26$ & $18,07 \pm 0,17$ & $4,64 \pm 0,12$ & $17,81 \pm 0,18$ & $8,27 \pm 0,06$ & $0,62 \pm 0,01$ \\
\hline Oléastre & $11,98 \pm 0,22$ & $8,73 \pm 0,17$ & $0,59 \pm 0,03$ & $10,04 \pm 0,42$ & $5,47 \pm 0,07$ & $0,15 \pm 0,01$ \\
\hline F1 $(8,2601)$ & $1597,323 * * *$ & $2115,661 * * *$ & $1229,675 * * *$ & 721,578*** & $1558,030 * * *$ & $1084,565 * * *$ \\
\hline F2 Variété $(8,81)$ & $109,963 * * *$ & $124,846 * * *$ & $94,767 * * *$ & $86,441 * * *$ & $187,410 * * *$ & $128,208 * * *$ \\
\hline F2 Arbre $(81,2610)$ & $27,158 * * *$ & $37,255 * * *$ & $21,861 * * *$ & $11,193 * *$ & $11,093 * *$ & $11,340 * *$ \\
\hline
\end{tabular}

Légende : F1, rapport de variance de l'analyse de variance à un seul critère (variété). F2, rapport de variance de l'analyse de variance à deux critères (variétés et arbres). Entre parenthèses le nombre de degrés de liberté. ***, significatif à $\mathrm{P}<0.001$ et $* *$ significatif à $\mathrm{P}<0.01$. Pour les codes caractères voir Tab. 2 . 
Ces différences correspondent à un gradient entre les fruits et noyaux de petite taille représentés par l'oléastre et ceux de grosse taille représentés par la variété d'olive de table Meslal. Mis à part la variété Asemlal qui se rapproche plus des petites tailles, les autres variétés présentent des tailles intermédiaires. Les variétés sélectionnées Menara et Haouzia présentent des valeurs de taille sensiblement similaires à celles des variétés locales Zeitoun, Hamrani, Bouchouk et Kortbi.

Ces données sont cohérentes avec celles obtenues dans une étude sur l'évaluation des variétés nationales et méditerranéennes cultivées au Maroc (Hadiddou et $a l ., 2013$ ) qui montrent un gradient similaire entre petite et grosse olives. Les petites olives correspondent à la variété Arbequine avec un poids de 1,3 g et une longueur de $13,7 \mathrm{~mm}$ ce qui lui donne une taille similaire à celle des olives de la variété Assemlal. Les olives de grandes tailles correspondent à celle des variétés Gordal Sevillana, Ascolona dura et Hojiblanca avec respectivement des olives de 6,2g, 5,5g et 4,0g. Les autres variétés ont des olives de masse intermédiaire comprise entre 2,3 et 3,7 g. D'autre part, une étude sur la caractérisation et l'identification d'écotypes locaux en Algérie portant sur 7 variétés locales a montré que ces variétés ont des olives de tailles nettement plus petites que celles des variétés du nord du Maroc (Abdessamad et al., 2018). En effet, la variété Limeli présente des olives de poids inférieur à $1 \mathrm{~g}(0,62 \mathrm{~g})$ comparables à l'oléastre alors que la variété Sigoise présente les plus grosses olives mais seulement avec une masse de 3,4g, c'est-à-dire des olives similaires à celles de la variété Zeitoun. Sigoise et Zeitoun sont des synonymes de la même variété dénommée Picholine Marocaine. De même, si on compare avec les variétés locales en Tunisie (Abaza et $a l ., 2002$ ) on trouve le même type de gradient avec : i) des variétés à petites olives dont la masse est inférieure ou égale à 1g (Zalmati, Chemlali et Oueslati), ii) des variétés à grosseur moyenne avec des olives à masse moyenne supérieure à $2 \mathrm{~g}$ (Seyali, Chetoui, Chemlali ou Garbaoui) et iii) des variétés à grosses olives comme Meski qui présente un poids moyen de 6,5 g. Cette dernière est présentée comme une variété à olive de table comparable à la variété Meslal du nord du Maroc. En ce qui concerne l'oléastre, Hannachi et al. (2008) ont étudié 78 écotypes en provenance d'agroécosystèmes et d'écosystèmes naturels. Les données relatives à la masse des fruits montrent un large intervalle de variation compris entre $0,21 \mathrm{~g}$ et $0,91 \mathrm{~g}$, la masse moyenne est inférieure à $1 \mathrm{~g}$. Donc, les fruits des oléastres tunisiens sont de taille comparable à celle des oléastres dans le nord du Maroc. D'autre part, il est intéressant de noter qu'il existe plusieurs variétés locales cultivées en Afrique du nord comme Limeli en Algérie, Zalmati, Chemlali et Oueslati en Tunisie ou Asemlal au Maroc qui présentent des olives de petites tailles, comparables aux olives de l'oléastre. Cette similitude va dans le sens des travaux réalisés sur la domestication et l'origine de l'olivier cultivé dans 
l'ouest de la méditerranée (Boucheffa et al., 2016, Khadari et al., 2018, Besnard et al., 2018).

\section{Caractères relatifs à la forme :}

Le rapport longueur sur largeur pour le fruit $(\mathrm{LlF})$ permet d'apprécier son allongement. En effet, plus ce rapport est nettement supérieur à 1, plus la forme est allongée. Au contraire, plus ce rapport est proche de 1, plus la forme est arrondie.

Pour les variétés étudiées, les valeurs obtenues pour ce caractère varient entre 1,27 et 1,45 (Tab. 4). On peut donc considérer que toutes les variétés ont des olives ovoïdes selon les normes du COI (1997). Les formes les plus allongées sont celles des olives des variétés Kortbi et Asemlal $(\mathrm{LlF}=1,45)$ et les olives les plus arrondies sont celles de la variété Meslal $(\mathrm{LlF}=1,28)$. Par contre, les noyaux montrent des formes nettement plus allongées que celles des olives (Tab. 4). Les variétés à noyaux les plus allongés sont Haouzia et Menara (LIN respectivement 2,16 et 2,11). Les noyaux d'oléastre tendent plus vers les formes ovoïdes $(\mathrm{LIN}=1,84)$ comme démontré par des analyses géométriques (Newton et al., 2014 ; Bourgeon et al., 2017). La hauteur de la plus grande largeur du fruit (HF) et du noyau (HN) et le rapport de la longueur sur la hauteur de la plus grande largeur (LHF et LHN) permettent d'affiner les différences de formes entre variétés. La forme est considérée comme elliptique si le rapport est sensiblement égal à 2 , ové s'il est inférieur à 2 ou obové s'il est supérieur à 2 . Les résultats obtenus montrent que la majorité des variétés ont des fruits plus ou moins elliptiques à légèrement obové (Tab. 4) car la hauteur de la plus grande largeur (HF) est généralement située au milieu de l'olive. La même constatation a été faite en ce qui concerne les noyaux. 
Tableau 4. Valeurs moyennes avec intervalle de confiance à $\mathrm{P}<0,05$ pour les caractères relatifs à la forme.

\begin{tabular}{lllllll} 
Variétés & HF & LIF & HN & LIN & LHF & LHN \\
\hline Zeitoune & $10,31 \pm 0,11$ & $1,32 \pm 0,01$ & $8,12 \pm 0,12$ & $2,03 \pm 0,02$ & $2,08 \pm 0,02$ & $2,02 \pm 0,02$ \\
\hline Hamrani & $11,19 \pm 0,15$ & $1,27 \pm 0,01$ & $7,87 \pm 0,13$ & $1,90 \pm 0,02$ & $2,06 \pm 0,05$ & $2,09 \pm 0,02$ \\
\hline Bouchouk & $11,89 \pm 0,15$ & $1,43 \pm 0,01$ & $8,45 \pm 0,15$ & $2,07 \pm 0,02$ & $1,83 \pm 0,02$ & $2,01 \pm 0,03$ \\
\hline Kortbi & $12,6 \pm 0,21$ & $1,45 \pm 0,01$ & $9,97 \pm 0,18$ & $2,01 \pm 0,02$ & $1,97 \pm 0,02$ & $1,94 \pm 0,02$ \\
\hline Meslal & $14,75 \pm 0,25$ & $1,28 \pm 0,01$ & $9,76 \pm 0,18$ & $1,99 \pm 0,02$ & $2,01 \pm 0,02$ & $2,16 \pm 0,03$ \\
\hline Assemlal & $7,51 \pm 0,38$ & $1,45 \pm 0,06$ & $6,96 \pm 0,39$ & $2,04 \pm 0,08$ & $2,02 \pm 0,07$ & $1,86 \pm 0,06$ \\
\hline Menara & $12,19 \pm 0,15$ & $1,35 \pm 0,01$ & $8,40 \pm 0,15$ & $2,11 \pm 0,02$ & $2,07 \pm 0,02$ & $2,15 \pm 0,03$ \\
\hline Haouzia & $12,41 \pm 0,16$ & $1,38 \pm 0,01$ & $8,37 \pm 0,11$ & $2,16 \pm 0,02$ & $2,01 \pm 0,01$ & $2,16 \pm 0,02$ \\
\hline Oléastre & $5,98 \pm 0,13$ & $1,38 \pm 0,01$ & $4,88 \pm 0,11$ & $1,84 \pm 0,08$ & $2,09 \pm 0,16$ & $2,08 \pm 0,08$ \\
\hline F1 $(\mathbf{8 , 2 6 0 1 )}$ & $\mathbf{1 0 4 4 , 5 1 3} * * *$ & $\mathbf{1 3 8 , 8 1 3} * * *$ & $\mathbf{4 2 8 , 5 4 8} * * *$ & $\mathbf{3 5 , 8 0 9} * * *$ & $\mathbf{7 , 7 9 2} * * *$ & $\mathbf{3 3 , 9 7 6} * * *$ \\
\hline F2 Variété $(\mathbf{8 , 8 1 )}$ & $\mathbf{8 3 , 2 7 4} * * *$ & $\mathbf{8 , 3 2 0} * * *$ & $\mathbf{4 0 , 3 5 0} * * *$ & $\mathbf{5 , 3 4 1} * *$ & $\mathbf{5 , 0 9 6} * *$ & $\mathbf{1 0 , 1 6 3} * *$ \\
\hline F2 Arbres $\mathbf{( 8 1 , \mathbf { 2 6 1 0 } )}$ & $\mathbf{2 1 , 2 8 4} * *$ & $\mathbf{3 6 , 2 1 7} * *$ & $\mathbf{1 5 , 9 2 1} * *$ & $\mathbf{8 , 3 8 1} * *$ & $\mathbf{1 , 3 3 7} \mathbf{n s}$ & $\mathbf{3 , 6 4 9} * *$ \\
\hline
\end{tabular}

Légende : F1, rapport de variance de l'analyse de variance à un seul critère (variété). F2, rapport de variance de l'analyse de variance à deux critères (variétés et arbres). Entre parenthèses le nombre de degrés de liberté. **, significatif à $\mathrm{P}<0.001$, ** significatif à $\mathrm{P}<0.01$ et ns, non significatif. Pour les codes caractères voir Tab. 2.

\section{Caractères relatifs à la teneur en huile :}

Parmi les caractères utilisés pour une estimation indirecte de la teneur en huile, c'est le pourcentage de la chair (PC) qui nous semble le plus informatif. Pour les deux autres rapports (MFMN et LFLN), ils traduisent également l'importance relative de la chair (mésocarpe) et donc indirectement la teneur en huile. Certes, ces caractères ne permettent pas de mesurer la teneur en huile, mais ils sont utiles pour comparer les potentialités de production d'huile des olives des différentes variétés étudiées. Nos résultats (Tab. 5) montrent que mis à part l'oléastre, Asemlal et dans une moindre mesure Kortbi, les autres variétés ont des pourcentages de chair supérieur à $80 \%$. En effet, les variations du pourcentage de chair entre les variétés ne sont pas très prononcées. Ainsi les variétés sélectionnées Haouzia et Menara ont des pourcentages similaires à Hamrani ou Zeitoun par exemple. Par contre, les autres rapports donnent des gradients de variations plus larges permettant de mieux différencier les variétés. Ainsi, en ce qui concerne la teneur en huile nos résultats montrent que les variétés locales des oliveraies traditionnelles du nord du Maroc y compris l'oléastre présentent un potentiel important. Kartas et al. (2016) dans une étude comparative entre variétés locales et sélectionnées dans la région d'Ouazzane située dans notre zone d'étude sont arrivés aux mêmes conclusions. 
Tableau 5. Valeurs moyennes avec intervalle de confiance à $\mathrm{P}<0,05$ pour les caractères relatifs à la productivité.

\begin{tabular}{llll} 
Variétés & PC & LFLN & MFMN \\
\hline Zeitoune & $0,84 \pm 0,002$ & $1,31 \pm 0,01$ & $6,50 \pm 0,10$ \\
\hline Hamrani & $0,86 \pm 0,003$ & $1,14 \pm 0,01$ & $7,19 \pm 0,50$ \\
\hline Bouchouk & $0,81 \pm 0,004$ & $1,29 \pm 0,01$ & $5,54 \pm 0,11$ \\
\hline Kortbi & $0,78 \pm 0,003$ & $1,29 \pm 0,01$ & $4,61 \pm 0,08$ \\
\hline Meslal & $0,87 \pm 0,002$ & $1,42 \pm 0,01$ & $8,05 \pm 0,18$ \\
\hline Assemlal & $0,75 \pm 0,03$ & $1,17 \pm 0,03$ & $4,36 \pm 0,47$ \\
\hline Menara & $0,87 \pm 0,008$ & $1,41 \pm 0,01$ & $7,63 \pm 0,14$ \\
\hline Haouzia & $0,86 \pm 0,002$ & $1,40 \pm 0,01$ & $7,48 \pm 0,12$ \\
\hline Oléastre & $0,71 \pm 0,01$ & $1,21 \pm 0,01$ & $3,81 \pm 0,13$ \\
\hline F1 Variété $(\mathbf{8 , 2 6 0 1 )}$ & $\mathbf{5 3 6 , 5 9 4} * * *$ & $\mathbf{4 0 3 , 0 6 6} * * *$ & $\mathbf{5 6 0 , 7 7 8} * * *$ \\
\hline F2 Variété $\mathbf{( 8 , 8 1 )}$ & $\mathbf{2 3 , 9 1 8 * * *}$ & $\mathbf{2 9 , 1 5 3} * * *$ & $\mathbf{3 5 , 6 6 2} * * *$ \\
\hline F2 Arbres $(\mathbf{8 1 , 2 6 1 0 )}$ & $\mathbf{8 6 , 2 6 2} * *$ & $\mathbf{2 4 , 8 6 9} * *$ & $\mathbf{3 1 , 8 7 1} * *$
\end{tabular}

Légende : F1, rapport de variance de l'analyse de variance à un seul critère (variété). F2, rapport de variance de l'analyse de variance à deux critères (variétés et arbres). Entre parenthèses le nombre de degrés de liberté. ***, significatif à $\mathrm{P}<0.001$, et $* *$ significatif à $\mathrm{P}<0.01$. Pour les codes caractères voir Tab. 2 .

\section{Polymorphisme et pouvoir discriminant des caractères mesurés}

L'analyse de la variance à un seul critère montre que l'ensemble des descripteurs utilisés ont un effet variété hautement significatif. En effet, les rapports de variance $(\mathrm{F} 1)$ sont tous hautement significatif. D'une manière générale, les caractères à rapports de variances les plus élevés peuvent être considérés comme les plus discriminants. Ainsi, les caractères relatifs à la taille seraient les plus discriminants (Tab. 3, 4 et 5). Par exemple pour le fruit, la largeur (IF) et la longueur (LF) et la masse (MF) montrent les F1 les plus élevés observés avec respectivement 2115,6 et 1597,3. Pour les descripteurs relatifs à la forme, mis à part la hauteur de la plus grande largeur (HF et HN), les autres descripteurs présentent les F1 les plus faibles comme par exemple le cas de LHN avec seulement 7,7. Il semble donc clair que la discrimination se caractérise essentiellement par un gradient de taille.

L'analyse de la variance à deux critères nous permet de tester deux niveaux de variabilité, le niveau "variété » en comparant les variétés entre elles et le niveau «intra variété » en comparant la variabilité entre les arbres de la même variété. La comparaison des deux niveaux de variabilité permet d'avoir une idée sur l'homogénéité et la stabilité des caractères utilisés pour la discrimination des variétés. Plus la variété est homogène et le caractère est stable, plus l'effet arbre sera moins important. Les résultats de l'analyse de variance à deux critères (Tab. 3, 4 et 5) confirment ceux obtenus par l'analyse de la variance à un seul critère car l'effet variété est hautement significatif pour l'ensemble des caractères étudiés. Cependant, même si les deux effets sont significatifs, l'effet variété présente des F2 nettement supérieures à ceux 
de l'effet arbre et cela pour la majorité des caractères étudiés. Ainsi, on peut estimer que même si la variabilité entre arbre de la même variété est significative, elle est moins importante que la variabilité observée entre variétés.

Tableau 6. Décomposition de la variance entre les facteurs Variété, Arbre et la résiduelle.

\begin{tabular}{|c|c|c|c|c|}
\hline \multicolumn{2}{|c|}{ Caractères } & Variété (\%) & Arbre (\%) & Résiduelle (\%) \\
\hline \multirow[t]{6}{*}{ Taille } & $\mathrm{LF}$ & 83,33 & 8,21 & 8,46 \\
\hline & $\mathrm{IF}$ & 86,74 & 7,61 & 5,65 \\
\hline & MF & 79,33 & 9,03 & 11,64 \\
\hline & $\mathrm{LN}$ & 69,76 & 8,32 & 21,92 \\
\hline & $\mathrm{MN}$ & 77,67 & 6,16 & 16,17 \\
\hline & IN & 83,36 & 4,53 & 12,11 \\
\hline \multirow[t]{6}{*}{ Forme } & $\mathrm{HN}$ & 57,27 & 15,24 & 27,49 \\
\hline & $\mathrm{HF}$ & 76,90 & 9,90 & 13,20 \\
\hline & LIN & 8,44 & 19,86 & 71,69 \\
\hline & $\mathrm{LlF}$ & 27,38 & 41,06 & 31,56 \\
\hline & LHF & 2,06 & 1,56 & 96,38 \\
\hline & LHN & 9,06 & 8,41 & 82,53 \\
\hline \multirow{3}{*}{$\begin{array}{c}\text { Teneur en } \\
\text { huile }\end{array}$} & $\mathrm{PC}$ & 61,11 & 29,48 & 9,41 \\
\hline & LFLN & 55,15 & 21,13 & 23,71 \\
\hline & MFMN & 63,07 & 19,75 & 17,19 \\
\hline
\end{tabular}

La décomposition de la variance (Tab. 6) confirme cette interprétation. En effet, il apparaît clairement pour tous les caractères mesurés relatifs à la taille que le niveau «variété » englobe la majorité de la variance avec des pourcentages supérieures à $70 \%$. Alors que pour les caractères relatifs à la forme seules les hauteurs de la plus grande largeur (HN et HF) expriment un pourcentage important au niveau «variété ». Pour les caractères en relation avec la teneur en huile la variation s'exprime au niveau « variété » mais avec des variances plus importantes au niveau « arbre».

\section{Structuration du polymorphisme et différenciation des variétés :}

La classification hiérarchique ascendante basée sur la similarité englobant les trois types de caractères mesurés a montré une bonne structuration des variétés étudiées (Fig. 4). 


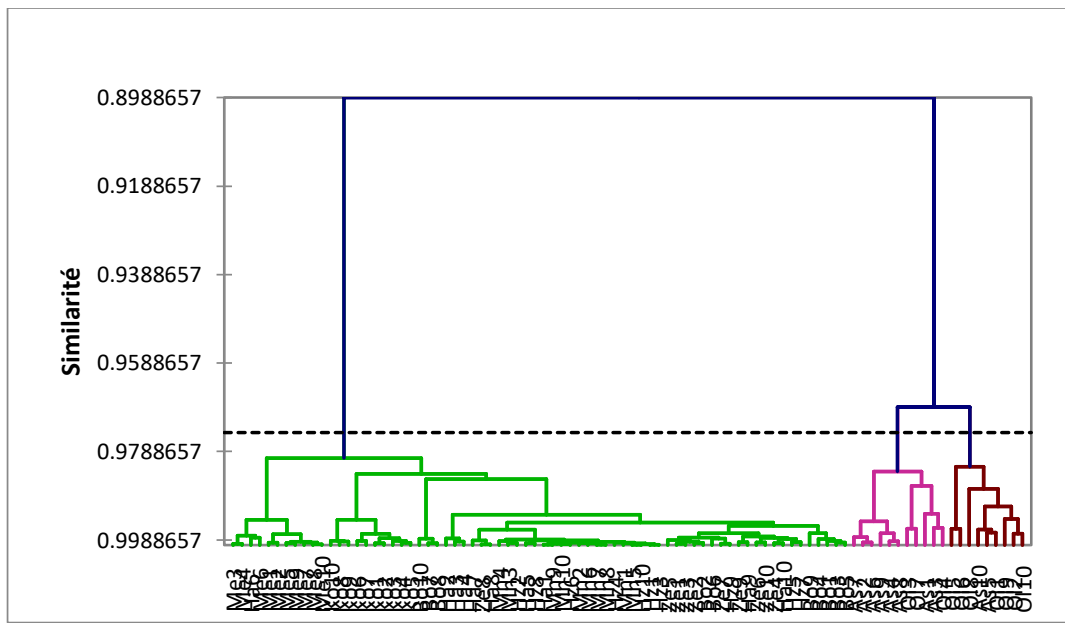

Figure 4. Dendrogramme de la classification hiérarchique. As: Asemlal, Bo: Bouchouk, Ha: Hamrani, Hz: Haouzia, Ko: Kortbi, Me: Meslal, Mn: Mnara, Ol: Oléastre et Ze: Zeitoun.

En effet, le dendrogramme montre deux groupes nettement distinct et qui opposent les variétés à fruit et noyau de petites tailles aux autres variétés. D'une part, le groupe avec les deux variétés à petites olives et noyaux correspondant à l'oléastre récolté dans un écosystème naturel et Asemlal, variété issus de semis et cultivée dans des agrosystèmes traditionnels. Cette dernière correspondrait à une forme férale (échappée des cultures et dispersée par semis) d'où la ressemblance avec l'oléastre. D'autre part, on retrouve l'ensemble des variétés cultivées multipliées végétativement. Parmi ces variétés la variété Meslal à olive de table se différencie nettement et constitue le pôle opposé aux variétés à petites olives. En plus de Meslal, la variété Kortbi se différencie nettement des autres variétés. Ainsi, on constate que les olives des variétés sélectionnées Haouzia et Menara ne permettent pas de les différencier des variétés locales du nord du Maroc. En effet, les olives de ces variétés présentent une forte similarité avec les variétés Zeitoun et Hamrani. Du point de vue génétique, la variété Zeitoun correspond à la variété Picholine marocaine dont le pool génétique engloberait les génotypes des variétés sélectionnées Haouzia et Menara et la variété Hamrani (Haouane, 2012, El Bakkali et al., 2013).

L'analyse factorielle discriminante a permis de vérifier la structuration mise en évidence par l'analyse hiérarchique. Le plan factoriel $(1,2)$ présente une bonne structuration de la variabilité avec $86 \%$ de la variance (Fig. 5). L'axe factoriel discriminant 1 englobe à lui seul $76,85 \%$ de la variance exprimée et correspond un gradient de taille des olives et noyaux opposant nettement les variétés à petite olives à la variété à olive de table Meslal (Fig. 5). Cet axe est défini par les variables de taille, de l'estimation de la teneur en huile et certains caractères de forme. Alors que l'axe factoriel discriminant 2 ne représente que $9,17 \%$ de la variance et il est expliqué par un petit nombre 
de caractères relatifs à la forme (LHF, LHN et LlF). Ainsi, on observe une nette différenciation le long de l'axe 1 selon un gradient de taille opposant du côté positif la variété Meslal à l'Oléastre et Asemlal du côté négatif de l'axe. Les autres variétés à olives et noyaux de tailles intermédiaires sont rassemblées au centre du graphe. Ce groupe présente un gradient de forme le long de l'axe 2 qui oppose du côté négatif la variété Kortbi aux variétés sélectionnées Menara et Haouzia. Les variétés Zeitoun, Bouchouk et Hamrani occupent une position intermédiaire et se chevauchent largement.

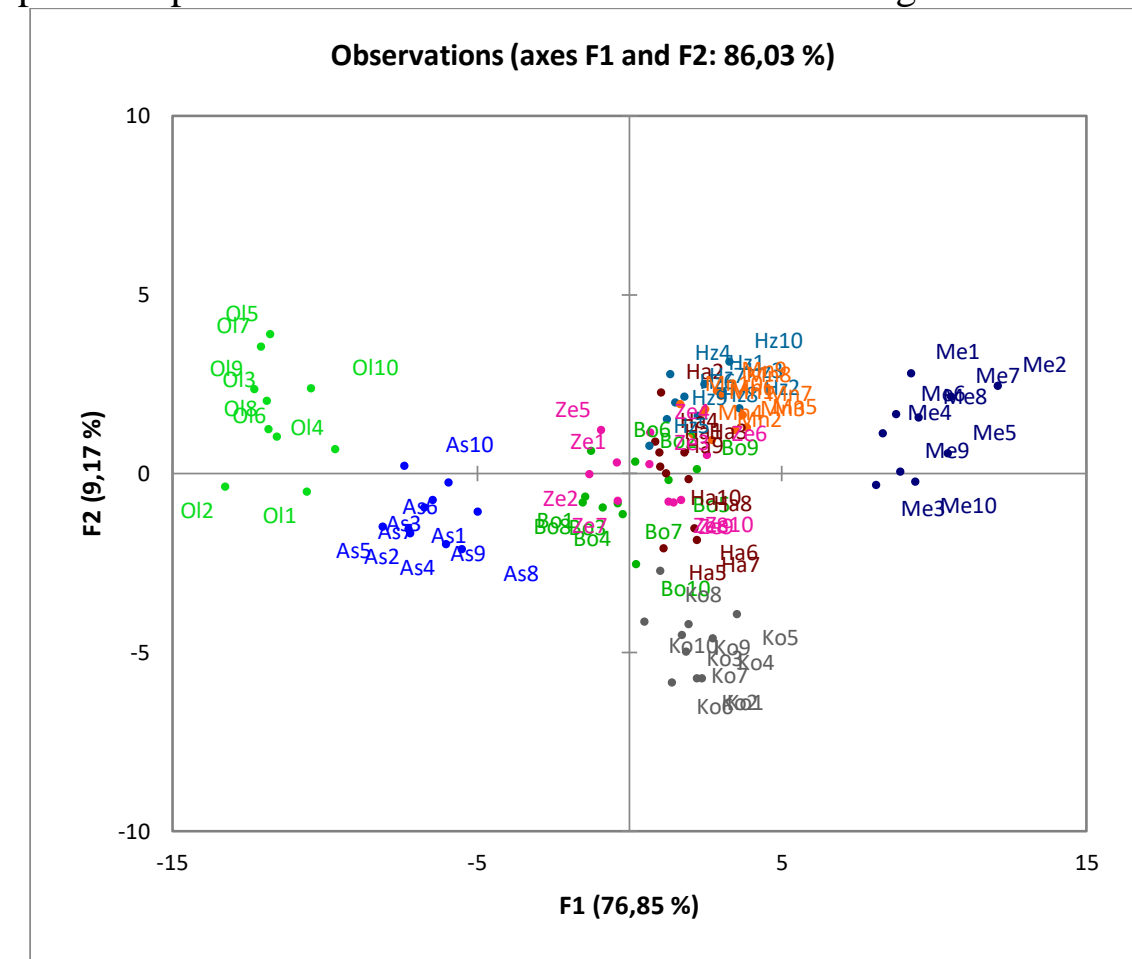

Figure 5. Projection des axes factoriels discriminant 1 et 2 de l'analyse factorielle discriminante.

\section{Conclusion}

Ce travail est une contribution importante pour la caractérisation des variétés locales négligées et sous-utilisées des oliveraies traditionnelles du Nord-Ouest du Maroc. La comparaison avec des variétés sélectionnées (Haouzia et Menara entre autres) et des variétés locales maghrébines a montré un important polymorphisme de ces variétés permettant de mettre en évidence leur potentiel et intérêt pour la sélection et l'amélioration de l'olivier. Les variétés locales à double usage des oliveraies traditionnelles du nord-ouest du Maroc présentent des olives de tailles nettement supérieures avec un intéressant potentiel pour la teneur en huile. En effet, nos résultats ont montré au niveau des descripteurs morphologiques quantitatifs relatifs à la taille et au 
potentiel en teneur d'huile une nette différenciation de ces variétés. Ainsi, nous avons mis en évidence un gradient de taille opposant les variétés à petite olives (Oléastre et Asemlal) à la variété Meslal à grosses olives. Les autres variétés ont des tailles relativement similaires et se différencient selon un deuxième gradient en relation avec des descripteurs secondaires relatifs à la forme des olives. Ce gradient oppose les deux variétés sélectionnées (Menara et Haouzia) à la variété Kortbi, alors que les autres variétés (Zeitoun, Hamrani et Bouchouk) se chevauchent largement. Cependant, on peut les différencier aisément par l'utilisation des caractères qualitatifs non pris en compte dans cette étude.

\section{Remerciements}

Cette étude a bénéficié de l'appui du projet « Laboratoire International Associé Evolea (INEE-CNRS /CNRST) ».

\section{References:}

1. Abdessemed, S., Abdessemed, A., Boudchicha, R. H. \& Benbouza H., 2018. Caractérisation et identification de quelques écotypes d'olivier Olea europaea L. en Algérie. Agriculture Journal. 8(2) :26-43p.

2. Abaza, L., Msallem, M., Daoud, D. \& Zarrouk, M. 2002. Caractérisation des huiles de sept variétés d'olivier tunisiennes. Oléagineux, Corps Gras, Lipides. Volume 9, n², pp. 174-9.

3. Adim, I. S. 2018. Analyse de la chaine des valeurs de l'Oléastre et son usage patrimonial. Master of Science. Institut Agronomique Méditerranéen de Montpellier. 107p.

4. Ater, M. \& Hmimsa, Y. 2013. Agrodiversité des agroécosystèmes traditionnels du pays Jbala (Rif, Maroc) et produits de terroirs. In Ilbert H., Tekelioglu Y., Çagatay S., Tozanli S. (eds.). Indications Géographiques, dynamiques socio-économiques et patrimoine bioculturel en Turquie et dans les pays méditerranéens. Montpellier : CIHEAM. pp.197-208.

5. Ater, M., Barbara, H. \& Kassout, J. 2016. Importance des variétés locales, de 1'oléastre et des pratiques traditionnelles de 1'oléiculture dans la région de Chefchaouen (Nord du Maroc). In Ater M., Essalouh L., Ilbert H., Moukhli A., Khadari B. (eds.). L'oléiculture au Maroc de la préhistoire à nos jours : pratiques, diversité, adaptation, usages, commerce et politiques. Montpellier : CIHEAM, 2016. 215 p.

6. Aumeeruddy-Thomas, Y., Hmimsa, Y., Ater, M. \& Khadari, B. 2014. Beyond the divide between wild and domesticated: spatiality, domesticity and practices pertaining to fig (Ficus carica L.) and olive (Olea europea L.) agroecosystems in Morocco. In: Eds Alex Chevalier, Elena Marinova, Leonor Peña-Chocarro, (eds.) « Crops and people: 
choices and diversity through time ». Earth EU, Brussels, OXFAM, London. pp.191-197.

7. Aumeeruddy-Thomas, Y. \& Caubet, D. 2017. «Savoirs paysans autour des huiles d'olive, (zaytun, Olea europaea var.europaea) et d'oléastre, (əl-bərri, Olea europaea var. sylvestris) Rif, nord du Maroc », Revue d'ethnoécologie [En ligne], Supplément 1 | 2017. URL : http://ethnoecologie.revues.org/3198;

DOI 10.4000/ethnoecologie.3198

8. Balaghi, R., Jlibene, M., Tychon, B. \& Eerens, H. 2013. Agrometeorological Cereal Yield Forecasting in Morocco. INRA, Morocco. 157p.

9. https://www.inra.org.ma/publications/ouvrages/prediction1113en.pdf

10. Besnard, G., Terral, J-F. \& Cornille, A. 2018. On the origins and domestication of the olive: a review and perspectives. Annals of Botany. doi: 10.1093/aob/mcx145, available online at www.academic.oup.com/aob.

11. Boucheffa, S., Miazzi, M. M., di Rienzo, V., Mangini, G., Fanelli, V., Tamendjari, A., Pignone, D. \& Montemurro, C. 2016. The coexistence of oleaster and traditional varieties affects genetic diversity and population structure in Algerian olive (Olea europaea L.) germplasm. Genet Resour Crop Evol. 64:379-390. DOI 10.1007/s10722-0160365-4

12. Boulouha, B., Loussert, R. \& Saadi, R. 1992. Étude de la variabilité phénotypique de la variété «Picholine Marocaine» dans la région du Haouz. Olivae 43:30-33p.

13. Bourgeon, O., Pagnoux, C., Mauné, S., García Vargas, E., Ivorra, S., Bonhomme, V., Ater, M., Moukhli, A. \& Terral, J.-F. 2017. Olive tree varieties cultivated for the great Baetican oil trade between the 1 st and the 4th centuries AD: morphometric analysis of olive stones from Las Delicias (Ecija, Province of Seville, Spain). Vegetation History and Archaeobotany, 27, 3, pp. 463-476.

14. Lauri, P., Barkaoui, K., Ater, M. \& Rosati A. 2019. Agroforestry for fruit trees in the temperate Europe and dry Mediterranean. In: Mosquera-Losada, M. R. and Prabhu, R. (ed.), Agroforestry for sustainable agriculture, Burleigh Dodds Science Publishing, Cambridge, UK; pp.385-418.

15. COI, 1997. Méthodologie pour la caractérisation primaire des variétés d'olivier. Projet RESGEN 9. 10p.

16. El Bakkali, A., Haouane, H., Hadiddou, A., Oukabli, A., Santoni, S., Udupa, S., Van Damme, P. \& Khadari, B. 2013. Genetic diversity of on-farm selected olive trees in Moroccan traditional olive orchards. Plant Genetic Resources, 11(2), pp.97-105. 
17. El Mouhtadi, I., Agouzzal, M. \& Guy, F. 2014. L’olivier au Maroc. OCL, 21(2), D203. Forecasting in Morocco. INRA, Morocco. 157p.

18. Hadiddou, A., Oukabli, A., Moudaffar, C., Mamouni, A., Gaboun, F., Mekaoui, A. \& El Fechtali, M. 2013. Evaluation des performances de production de 14 variétés d'olivier (Olea europaea L.) Nationales et méditerranéennes dans deux systèmes contrastés de culture (pluvial et irrigué) au Maroc. Al Awamia, 127, pp.22-43.

19. Hannachi, H., Breton, C., Msallem, M., El Hadj, S. B., El Gazzah, M. \& Bervillé, A., 2008. Differences between native and introduced olive cultivars as revealed by morphology of drupes, oil composition and SSR polymorphisms: a case study in Tunisia. Scientia Horticulturae, 116(3), pp.280-290.

20. Haouane, H. 2012. Origines, domestication et diversification variétale chez l'olivier (Olea europaea L.) à l'ouest de la Méditerranée. Thèse (Dr. Evolution, Ecologie, Ressources Génétiques, Paléontologie) : Montpellier Supagro, Université de Marrakech. 272 p.

21. Hmimsa, Y. \& Ater, M. 2008. Agrodiversity in the traditional agrosystems of the Rif mountains (North of Morocco). Biodiversity, vol. $9, \mathrm{n}^{\circ} 1-2$, pp.78-81.

22. Kartas, A. 2017. Caractérisation morphologique et évaluation des potentialités et performances agronomiques et physico-chimiques des variétés introduites et des types locaux d'olivier (Olea europaea L.) cultivés dans la région d'Ouazzane (Nord du Maroc). Thèse de Doctorat National, Faculté des Sciences, Université Ibn Tofail, 193p

23. Kartas A., Chliyeh M., Ouazzani Touhami A., Gaboun F., Benkirane R. \& Douira A., 2015. Diversity of the olive tree (Olea europaea L.) genetic resources in the region of Ouazzane (North of Morocco): characterization by using the morphological descriptors. Int. J. Pure App. Biosci. 2 (4): 10-21.

24. Kartas A., Chliyeh M., Touati J., Ouazzani Touhami A., Gaboun F., Benkirane R. \& Douira A., 2016. Evaluation of bio-agronomical characteristics of olive fruits (Olea europaea L.) of the introduced varieties and local types grown in the Ouazzane areas (Northern Morocco). International Journal of Advances in Pharmacy, Biology and Chemistry, 5(1):39-43.

25. Khadari, B., Charafi, J., Moukhli, A. \& Ater, M. 2008. Substantial genetic diversity in cultivated Moroccan olive despite a single major cultivar: a paradoxical situation evidenced by the use of SSR loci. Tree Genetics \& Genomes, vol. 4, n'2, pp. 213-221. http://dx.doi.org/10.1007/s11295-007-0102-4.

26. Khadari, B. \& El Bakkali, A. 2018. Primary Selection and Secondary Diversification: Two Key Processes in the History of Olive 
Domestication. International Journal of Agronomy. Volume 2018, Article ID 5607903, https://doi.org/10.1155/2018/5607903

27. Kradi, C. 2012. L'agriculture solidaire dans les écosystèmes fragiles au Maroc. Rabat : Editions INRA. 185p. http://www.inra.org.ma/publications/ouvrages/kradi1201.pdf

28. MAPM, (Ministère de l'Agriculture et de la Pêche Maritime). 2008. Plan Maroc vert : premières perspectives sur la stratégie agricole. Rabat : MAPM. 28 p.

29. Moukhli, A., Essalouh, L., El Bakkali, A., El Modafar, C. \& Khadari, B. 2016. Eclairage sur l'origine de la Picholine marocaine. In : Ater M., Essalouh L., Ilbert H., Moukhli A., Khadari B., (éds). L'oléiculture au Maroc : de la préhistoire à nos jours : pratiques, usages, commerce et politiques. Montpellier : CIHEAM. pp. 79-92.

30. Moukhli, A., Haouane, H., El Modafar, C. \& Khadari, B. 2013. Histoire de l'introduction et de la diffusion de l'oléiculture au Maroc. In: Ilbert H., Tekelioglu Y., Çagatay S., Tozanli S. (eds.). Indications Géographiques, dynamiques socio-économiques et patrimoine bioculturel en Turquie et dans les pays méditerranéens. Montpellier : CIHEAM. pp.169-196.

31. Newton, C., Lorre, C., Sauvage, C., Ivorra, S. \& Terral, J.-F. 2014. On the origins and spread of Olea europaea L. (olive) domestication: evidence for shape variation of olive stones at Ugarit, Late Bronze Age, Syria - a window on the Mediterranean Basin and on the westward diffusion of olive varieties. Vegetation History and Archaeobotany, 23(5), pp. 567-575. 